\title{
UNSTABLE TOWERS IN THE ODD PRIMARY HOMOTOPY GROUPS OF SPHERES
}

\author{
BY
}

\author{
MARTIN BENDERSKY
}

\begin{abstract}
The unstable elements in filtration 2 of the unstable Novikov spectral sequence are computed. These elements are shown to survive to elements in the homotopy groups of spheres which are related to Im $J$. The computation is applied to determine the Hopf invariants of compositions of $\operatorname{Im} J$ and the exponent of certain sphere bundles over spheres.
\end{abstract}

1. Introduction. Let $p$ be an odd prime and $\hat{S}^{2 m}$ a space with $H^{*}\left(\hat{S}^{2 m} ; Z_{p}\right) \simeq$ $P\left(\iota_{2 m}\right) / \iota^{p}$. There are EHP sequences relating $\hat{S}^{2 m}$ and the odd spheres [17]:

$$
\begin{aligned}
& \rightarrow \pi_{i}\left(S^{2 m-1}\right) \stackrel{\sigma}{\rightarrow} \pi_{i+1}\left(\hat{S}^{2 m}\right) \stackrel{H}{\rightarrow} \pi_{i+1}\left(S^{2 m p-1}\right) \stackrel{P}{\rightarrow} \pi_{i-1}\left(S^{2 m-1}\right) \rightarrow, \\
& \rightarrow \pi_{i}\left(\hat{S}^{2 m}\right) \stackrel{\sigma}{\rightarrow} \pi_{i+1}\left(S^{2 m+1}\right) \stackrel{H^{\prime}}{\rightarrow} \pi_{i+1}\left(S^{2 m p+1}\right) \stackrel{P^{\prime}}{\rightarrow} \pi_{i-1}\left(\hat{S}^{2 m}\right) \rightarrow
\end{aligned}
$$

Let $\alpha_{k} \in \pi_{2 m p+2(p-1) k}\left(S^{2 m p+1}\right)$ be an element in $\operatorname{Im} J$ of order $p$. From [17] $H P^{\prime}\left(\alpha_{k}\right)=p \alpha_{k}=0$, and $P^{\prime}\left(\alpha_{k}\right)$ desuspends to the $(2 m-1)$ sphere. In this paper we consider the problem of computing the maximal desuspensions of these elements to an odd sphere and their Hopf invariants on their spheres of origin. The 2 primary answer is described in [13]. Our methods are somewhat different. We simply compute the unstable elements in filtration 2 of the unstable Novikov spectral sequences and with a helping hand from Cohen, Moore and Neisendorfer [7], and Selick [16] we deduce the survival of these elements and prove there are no extensions. Because the odd primary desuspensions of $\operatorname{Im} J$ are more regular than for $p=2$ we obtain a rather simple picture for the desuspension pattern.

The following is proven in Theorem (3.2). Let the stem be equal to $2 s p^{t}(p-1)-2$ ( $s$ prime to $p$ ); then we have the following chart.

Explanation. An unstable element is born on $S^{3}$. It maps to $p$ times a generator on $S^{5}$. The pattern of generator suspending to $p$ times a generator and the kernel of the double suspension being zero persists to $S^{2 t+3}$, where a $Z_{p^{t+1}}$ is generated. For $S^{2 j+1}, t+1<j<s p^{t}-t$, the double suspension is given by $Z_{p^{t+1}} \stackrel{x p}{\rightarrow} Z_{p^{t+1}}$. From $S^{2\left(s p^{t}-t\right)-1}$ on the double suspension is the map $Z_{p^{i+1}} \rightarrow Z_{p^{i}}$ which sends a generator to a generator. In $\$ 4$ we apply the results of $\$ 3$ to some questions posed by $\mathrm{J}$. Neisendorfer. We first identify compositions of $\operatorname{Im} J$ in the unstable two-line.

Received by the editors July 22, 1983 and, in revised form, January 30, 1984.

1980 Mathematics Subject Classification. Primary 55E40, 55H25, 55B20. 


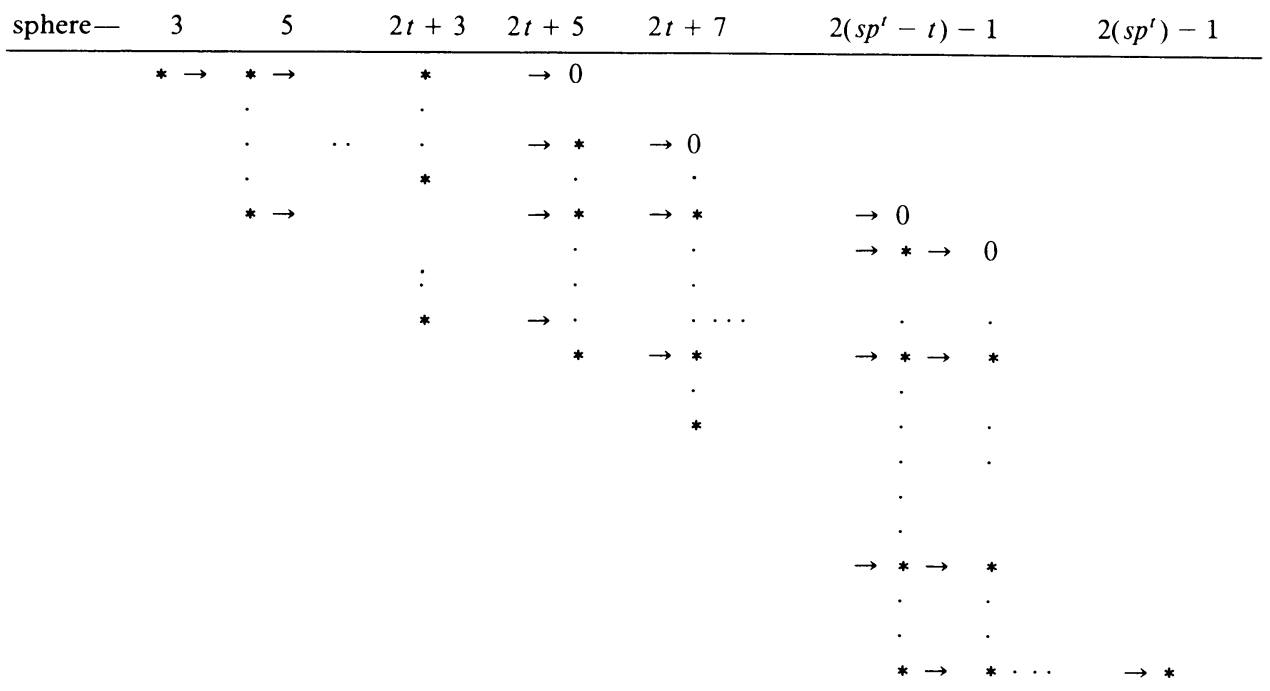

Arithmetic now shows that the only decomposable mod $p$ Whitehead product is $\alpha_{1}^{2}$, and, if $p=3, \alpha_{2} \alpha_{1}$. This takes advantage of the low filtration of the Whitehead product (this has also been proven by B. Gray using secondary cohomology operations). On the other hand, the low filtration of $P(\operatorname{Im} J)$ has the disadvantage of not guaranteeing that $\alpha_{i} \alpha_{j}$ vanish in homotopy when they do in Ext. As a final application we show that the spaces $B_{n}(p)$ [15] have maximal Ext exponent if $n=1$. If $p=3$ we can conclude the same for homotopy. We are also able to resolve some undecided cases in the computations of [15].

We use the notation $Z_{(p)}$ and $Z_{p}$ for the integers localized at $p$ and the integers $\bmod p$, respectively.

$$
\begin{gathered}
x \\
\vdots \\
y
\end{gathered}
$$

denotes an extension and $\Sigma^{r}$ the $r$-fold suspension. For any ring $A, A(n)$ is the free, left $A$-module on a generator of dimension $n$. If $\eta_{R}: B P_{*} \rightarrow B P_{*} B P$ is the right action we write $\gamma \cdot v$ for $\eta_{R}(v) \gamma$ with $v \in B P_{*}, \gamma \in B P_{*} B P . \nu_{p}(k)$ is defined by $k=p^{\nu_{p}(k)} a,(a, p)=1$.

The author would like to thank J. Neisendorfer for his many helpful conversations and his interest in this project. B. Gray has obtained some of the above results using different methods [18].

2. The unstable Novikov spectral sequence. For a simply connected space $X$ there is a spectral sequence (the unstable Novikov spectral sequence) which converges to the homotopy groups of $X$ localized at $p$ with the $E_{2}$ term depending on the $B P$ 
homology of $X[5,2,3]$. The elements described in $\$ 1$ appear as unstable elements in filtration 2 of this spectral sequence. In order to describe the $E_{2}$ term we recall [2, (2.13)]. We assume some familiarity with [1, 5 and 3] and the reader is referred to these papers for details. The unstable generators for $B P_{*}$ and $\Gamma=B P_{*} B P$ are described in $[3, \S 3]$ :

$$
A=B P_{*} \simeq Z_{(p)}\left[u_{1}, u_{2}, \ldots\right]
$$

where the $u_{i}$ are the Araki generators (denoted $v_{i}$ in [3]), and

$$
\Gamma \simeq B P_{*}\left[h_{1}, h_{2}, \ldots\right]
$$

where $h_{i}=c\left(t_{i}\right), c$ the canonical anti-isomorphism of $\Gamma$.

Definition $(2.1)[3,2.13]$. If $M$ is a nonnegatively graded free left $B P_{*}$-module, $U(M)$ is defined to be the $B P_{*}$ span of

$$
\left\{h^{I} \otimes m \mid 2\left(i+i_{2}+\cdots\right)<\text { degree } m\right\} \subset \Gamma \otimes_{A} M .
$$

For an arbitrary $B P_{*}$-module $M$ let $F_{1} \stackrel{f}{\rightarrow} F_{0} \rightarrow M \rightarrow 0$ be exact with $F_{i}$ free, and define $U(M)$ to be the cokernel of $U(f)$.

Suppose we are given an unstable $\Gamma$-comodule $M[5$, p. 240] with coaction $\psi$ : $M \rightarrow U(M)$. Then the unstable cobar complex is the chain complex $C^{s, t}(M) \simeq$ $U^{s}(M)$ with differential given by

$$
\begin{aligned}
d\left(\left[\gamma_{1}\left|\gamma_{2}\right| \cdots \mid \gamma_{s}\right] m\right)= & {\left[1\left|\gamma_{1}\right| \cdots \mid \gamma_{s}\right] m+\sum_{j=1}^{s}(-1)^{j}\left[\gamma_{1}|\cdots| \gamma_{j}^{\prime}\left|\gamma_{j}^{\prime \prime}\right| \cdots \mid \gamma_{s}\right] m } \\
& +(-1)^{s+1} \sum\left[\gamma_{1}|\cdots| \gamma_{s} \mid \beta_{i}\right] m_{i}
\end{aligned}
$$

where $\gamma_{1}, \ldots, \gamma_{s} \in \Gamma, \psi\left(\gamma_{j}\right)=\sum \gamma_{j}^{\prime} \otimes \gamma_{j}^{\prime \prime}$ and $\psi(m)=\sum \beta_{i} \otimes m_{i}$ [5, p. 244]. We define the unstable Ext group to be the homology of the unstable cobar complex

$$
\operatorname{Ext}_{\mathscr{\mathscr { U }}}^{s, t}(M) \simeq H^{s, t}(C(M))
$$

If $X$ is a simply connected complex with $H^{*}\left(X ; Z_{(p)}\right)$ a free algebra, then we may identify the $E_{2}$ term of the unstable Novikov spectral sequence for $X[5, \S 7]$

$$
E_{2}(X) \simeq \operatorname{Ext}_{\mathscr{Q}}\left(P B P_{*} X\right)
$$

where $P$ denotes the primitives.

If $H^{*}\left(X ; Z_{(p)}\right)$ is torsion-free but not a free algebra (e.g. $\left.X=\hat{S}^{2 m}\right)$, we may give an algebraic description of $E_{2}(X)$ as an Ext in a nonabelian category. Fortunately we shall not need an explicit description of this Ext and simply write $E_{2}(X)$ for such spaces. 
The EHP sequences described in $\$ 1$ appear at the $E_{2}$ level of the unstable Novikov spectral sequence $[3,6]$. Specifically we have the commutative diagram of exact sequences:

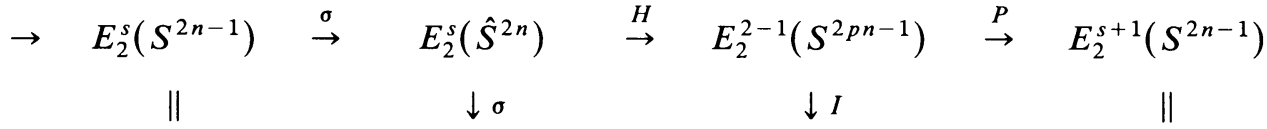

$$
\begin{aligned}
& \rightarrow E_{2}^{s}\left(S^{2 n-1}\right) \quad \stackrel{\sigma^{2}}{\rightarrow} \quad E_{2}^{s}\left(S^{2 n+1}\right) \quad \stackrel{H_{2}}{\rightarrow} \operatorname{Ext}^{s-1}(W(n)) \stackrel{P_{2}}{\rightarrow} \quad E_{2}^{s+1}\left(S^{2 n-1}\right) \\
& \downarrow H^{\prime} \\
& E_{2}^{s}\left(S^{2 p n+1}\right) \\
& \downarrow P^{\prime} \\
& E_{2}^{s+1}\left(\hat{S}^{2 n}\right)
\end{aligned}
$$

TheOREM (2.4) [3]. (i) $W(n) \simeq B P_{*} / p\left\{x_{2 p n-1}, x_{2 p^{2}-1}, \ldots\right\}$. The unstable $\Gamma$-comodule structure of $W(n)$ is given by

$$
\psi\left(x_{2 p^{k} n-1}\right)=\sum_{i} p^{k-i} h_{k-i}^{n p^{i}} \otimes x_{2 p^{i} n-1} .
$$

(ii) If $z \in \operatorname{Ext}_{\mathscr{U}}(W(n))$ is represented in the unstable cobar complex by $\sum \gamma_{k} \otimes$ $x_{2 p^{k} n-1}$, then

$$
P_{2}(z)=d\left(\sum \gamma_{k} \otimes p^{k-1} h_{k}^{n}\right) \otimes \iota_{2 n-1} .
$$

(iii) Every element $x \in E_{2}^{s}\left(S^{2 n+1}\right)$ may be represented in the unstable cobar complex by a cycle of the form

$$
\sum \gamma_{k} \otimes p^{k-1} h_{k}^{n} \otimes \iota_{2 n+1}, \quad \gamma_{k} \in C^{*}\left(A\left(2 p^{k} n-1\right) \otimes Z_{p}\right)
$$

mod terms which desuspend. $\left(A\left(2 p^{k} n-1\right)\right.$ denotes a free $B P_{*}$-module on a generator ı of dimension $2 p^{k} n-1$.) Furthermore $H_{2}(X)=\sum \gamma_{k} \otimes x_{2 p^{k} n-1}$.

(iv) $I$ is the composite

$$
E_{2}\left(S^{2 p n-1}\right) \simeq \operatorname{Ext}_{\mathscr{U}}(A(2 p n-1)) \rightarrow \operatorname{Ext}_{\mathscr{U}}(W(n)),
$$

where the second map is the mod $p$ reduction to the bottom class of $W(n)$.

For convenience we shall say a class $x \in E_{2}\left(S^{2 n+1}\right)$ desuspends to the $(2 k+1)$ sphere (or to $\hat{S}^{2 k}$ ) if it does so in $E_{2}$.

In a similar way we say a class dies on the $(2 m+1)$ sphere if it does so in $E_{2}$.

It is important to note that the coaction (2.5) is unstable, i.e. $\Sigma^{2} \psi\left(x_{2 p^{k} n-1}\right)=1 \otimes$ $x_{2 p^{k} n-1}$. For example, the term $p h_{1}^{n p^{i}} \otimes x_{2 p^{i} n-1}$ is in $U(W(n))$ and is not zero $\bmod p$ $[3,3.6]$ but $p h_{1}^{n p^{i}} \otimes x_{2 p^{i} n+1}$ is zero.

We now restrict $p$ to be odd.

The elements in filtration 1 all survive to $E_{\infty}$ and desuspend in $E_{2}$ exactly as far as they do in homotopy [5, (9.16)]. Let $\alpha_{k / j} \in \operatorname{Ext}_{\Gamma}^{1,2(p-1) k}\left(B P_{*}, B P_{*}\right)$ denote the stable element in filtration 1 of order $p^{j}, j \leqslant \nu_{p}(k)+1$. Then $\alpha_{k / j}$ desuspends in $E_{2}$ 
and in homotopy to the $(2 j+1)$ sphere [9]. For $\alpha_{k / j} \in E_{2}\left(S^{2 j+1}\right)$,

$$
H_{2}\left(\alpha_{k / j}\right)=u_{1}^{k-j}, \quad H^{\prime}\left(\alpha_{k / j}\right)=\alpha_{k-j} \quad\left(\alpha_{k-j}=\alpha_{k-j / 1}\right) .
$$

In particular when $P^{\prime}\left(\alpha_{k / j}\right) \in \pi_{2 p(m+k)-2(k-j)}\left(\hat{S}^{2 m}\right)$ is nonzero it is detected in filtration 2 of the unstable Novikov spectral sequence for $\hat{S}^{2 m}$. We shall need the unstable cobar names for $\alpha_{k / j}=d\left(u_{1}^{k}\right) / p^{j}$.

Proposition (2.8). (i) If $m \geqslant j$,

mod terms defined on $S^{2 j-1}$.

$$
\alpha_{k / j} \otimes \iota_{2 m+1}=u_{1}^{k-j} h_{1}^{j} \otimes \iota_{2 m+1}
$$

(ii) If $m \geqslant \nu+2\left(\nu=\nu_{p}(k)\right)$, then

$$
\alpha_{k / \nu+1} \otimes \iota_{2 m+1}=h_{1} u_{1}^{k-1} \otimes \iota_{2 m+1} \bmod p .
$$

Proof. (i) is proven in [5, (9.2)]. To prove (ii) we examine

$$
-d\left(u_{1}^{k}\right) / p^{\nu+1}=\sum_{j=1}^{k}(-1)^{j}\left(\begin{array}{l}
k \\
j
\end{array}\right) p^{j-\nu-1} h_{1}^{j} \cdot u_{1}^{k-j} \otimes \iota_{2 m+1} .
$$

If $j>\nu+1, p^{j-\nu-1} h_{1}^{j} \otimes \iota_{2 m+1}$ is $0 \bmod p$ in $U(A(2 m+1))$. For $1<j \leqslant \nu+1$, $p^{\nu+2-j} \mid\left(\begin{array}{c}k \\ j\end{array}\right)[14,(8.21)]$. So

$$
\left(\begin{array}{l}
k \\
j
\end{array}\right) p^{j-\nu-1} h_{1}^{j} \otimes \iota_{2 m+1}=a p h_{1}^{j} \otimes \iota_{2 m+1}=0
$$

in $U(A(2 m+1))$.

Composition appears at the $E_{2}$ level with order reverse to the usual functional composition in homotopy. For convenience we shall write composition in homotopy in the same order as it appears in Ext. In order to simplify notations we introduce the following terminology. If $\alpha \in \pi_{k}\left(s^{n}\right)$ we say $\beta$ is an $r$-fold suspension on $\alpha$ in homotopy if $\beta \in \pi_{t}\left(S^{k}\right)$ (so $\beta \alpha$ is defined) and $\beta=\sum^{r} \gamma$ for some $\gamma \in \pi_{t-r}\left(S^{k-r}\right)$, i.e. $\beta$ is an $r$-fold suspension on the domain sphere of $\alpha$. If $x \in C^{*}(A(n))$ then we say $y=\gamma_{1} \otimes \gamma_{2} \otimes \cdots \otimes \gamma_{i}\left(\gamma_{j} \in \Gamma\right)$ is an $r$-fold suspension in Ext on $x$ if $y \otimes x \in$ $C^{*}(A(n))$ and $y \in C^{*}(A(|x|+n-r))$, where $|x|$ is the homological dimension of $x$. Of course we do not require $y$ or $x$ to survive to homotopy; however, even when both $x$ and $y$ are permanent cycles the number of suspensions in homotopy differs from the number of suspensions in Ext. For example, with $p=3, \alpha_{9 / 3}$ has 0 suspensions with respect to $\alpha_{1} \otimes \iota_{3}$ in Ext, but the composition $\alpha_{9 / 3} \alpha_{1}$ is not even defined if $\alpha_{1} \in \pi_{6}\left(S^{3}\right)$.

3. Unstable towers. Throughout this section $p$ is a fixed odd prime. In order to state our main theorem we recall the notation of [3].

Definition (3.1). $\left\{\alpha_{n} h_{1}^{m}\right\}_{h_{1}^{m+n}}$ denotes the tower

$$
\begin{gathered}
\left(\tilde{\alpha}_{m+n-1} h_{1}\right)_{u_{1}^{m-1} h_{1}^{n+1}} \\
\vdots \\
\left(\tilde{\alpha}_{n+1} h_{1}^{m-1}\right)_{u_{1} h_{1}^{m+n-1}} \\
\vdots \\
\left(\tilde{\alpha}_{n} h_{1}^{m}\right)_{h_{1}^{n+m}}
\end{gathered}
$$


where $\tilde{\alpha}_{k}$ is the generator of the stable $J$ homomorphism in the $(2(p-1) k-1)$ stem. $\left(\tilde{\alpha}_{k} h_{1}^{r}\right)_{u_{1}^{a} h_{1}^{\prime}}$ denotes an element born in Ext on the $(2 r+1)$ sphere which dies or is made homologous to an element higher in the tower on the $(2 t+1)$ sphere.

Before proceeding it is important to be aware of the abuses in the above notation. $\tilde{\alpha}_{k} h_{1}^{r}$ is only meant to suggest that the coefficient of $h_{1}^{r}$ is $h_{1} u_{1}^{k-1}$ (i.e. the stable name for $\tilde{\alpha}_{k} \bmod p$ ). In fact, we require 2 suspensions of $\tilde{\alpha}_{k}$ with respect to $h_{1}^{r} \otimes \iota_{2 r+1}$ in order to assert $H_{2}\left(\tilde{\alpha}_{k} h_{1}^{r} \otimes \iota_{2 r+1}\right)=\tilde{\alpha}_{k}$.

THEOREM (3.2). The unstable elements in filtration 2 of the unstable Novikov spectral sequence at an odd prime $p$ are described as follows:

(i) In stems $\not \equiv-2(\bmod 2(p-1))$, Ext $=0$.

(ii) In stem $2(p-1) s p^{n}-2(s \neq 0 \bmod p)$, the unstable elements in filtration 2 are given by the tower $\left\{\alpha_{n+1} h_{1}^{s p^{n}-n-1}\right\}_{h_{1}^{s p^{n}}}$.

(iii) If $\tilde{\alpha}_{n+r}=\sum^{2} \gamma$ for $\gamma \in \operatorname{Ext}_{\mathscr{U}}^{1}\left(A\left(2 p\left(s p^{n}-n-r\right)-1\right)\right)$, then $H_{2}\left(\tilde{\alpha}_{n+r} h_{1}^{s p^{n}-n-r}\right)=\tilde{\alpha}_{n+r}$. In this case $\tilde{\alpha}_{n+r} h_{1}^{s p^{n}-n-r}$ is born on $\hat{S}^{2\left(s p^{n}-n-r\right)}$ with $H\left(\tilde{\alpha}_{n+1} h_{1}^{s p^{n}-n-r}\right)=\tilde{\alpha}_{n+1} \bmod p$.

(iv) If $\tilde{\alpha}_{n+r} \neq \sum^{2} \gamma$ for any $\gamma$ as in (iii), then

$$
H_{2}\left(\tilde{\alpha}_{n+r} h_{1}^{s p^{n}-n-r}\right)=h_{1} u_{1}^{n+r-1} x_{2 p\left(s p^{n}-n-r\right)-1} .
$$

In this case $\tilde{\alpha}_{n+r} h_{1}^{s p^{n}-n-r}$ is born on $S^{2\left(s p^{n}-n-r\right)+1}$.

(v) $\left\{\alpha_{n+1} h_{1}^{s p^{n}-n-1}\right\}_{h_{1}^{p^{n}}}$ survives to $E_{\infty}$ and there are no higher Novikov extensions.

EXAMPLE (3.3). We illustrate (3.2)(iv) and (v) with some examples for $p=3$. Our

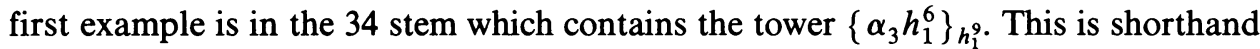
for the following elements in $E_{2}$ :

$$
\begin{array}{ccccccccc}
S^{3} & S^{5} & S^{7} & S^{9} & S^{11} & S^{13} & S^{15} & S^{17} & S^{19} \\
\alpha_{8} \alpha_{1} & \alpha_{8} \alpha_{1} & \alpha_{8} \alpha_{1} & \rightarrow 0 & & & & & \\
& \vdots & \vdots & & & & & & \\
& \alpha_{7} h_{1}^{2} & \alpha_{7} h_{1}^{2} & \alpha_{7} h_{1}^{2} & \rightarrow 0 & & & & \\
& \vdots & \vdots & & & & & \\
& \tilde{\alpha}_{6} h_{1}^{3} & \tilde{\alpha}_{6} h_{1}^{3} & \tilde{\alpha}_{6} h_{1}^{3} & \rightarrow 0 & & & \\
& & \vdots & \vdots & & & & \\
& & \alpha_{5} h_{1}^{4} & \alpha_{5} h_{1}^{4} & \alpha_{5} h_{1}^{4} & \rightarrow 0 & & \\
& & & \vdots & \vdots & & & \\
& & & \alpha_{4} h_{1}^{5} & \alpha_{4} h_{1}^{5} & \alpha_{4} h_{1}^{5} & \rightarrow 0 & \\
& & & & \vdots & \vdots & & \\
& & & & \tilde{\alpha}_{3} h_{1}^{6} & \tilde{\alpha}_{3} h_{1}^{6} & \tilde{\alpha}_{3} h_{1}^{6} & \rightarrow 0
\end{array}
$$

The survival of these elements is an induction starting with a proof that $\tilde{\alpha}_{3} h_{1}^{6}$ on $S^{13}$ survives. We inductively assume for $i \geqslant 6$ that the generator $\lambda_{i}$ on $S^{2 i+1}$ survives. $p \lambda_{i}$ must double desuspend in homotopy by [7]. The only element in Ext which can detect it is $\lambda_{i-1}$, the generator on $S^{2 i-1}$. To show there are no higher $B P$ 
extensions we first note that the elements on $S^{3}, S^{5}$ and $S^{7}$ cannot have any higher extension by [7 and 16]. From $S^{9}$ on, the elements of order $p$ in $E_{2}$ will be shown to survive to elements in the kernel of the double suspension map in homotopy and therefore have order $p$ in homotopy by [7]. Hence there cannot be any higher $B P$ extensions.

Our next example is the tower in the 38 stem. The element $\tilde{\alpha}_{9} h_{1} \otimes \iota_{3}$ satisfies the condition of (iv). $\tilde{\alpha}_{9}=\alpha_{9 / 3}$ is not a double suspension in Ext on $h_{1} \otimes \iota_{3}$. We have $H_{2}\left(\tilde{\alpha}_{9} h_{1}\right)=h_{1} u_{1}^{8} \otimes x_{5}$. If $\tilde{\alpha}_{9} h_{1}$ desuspended to $\hat{S}^{2} h_{1} u_{1}^{8} \otimes x_{5}$, would have to be in the image of $I$ (see (2.3)). However, $\beta\left(h_{1} u_{1}^{8} \otimes x_{5}\right)=\alpha_{1} \alpha_{8} \otimes \iota_{5}(\beta$ is the Bockstein homomorphism associated to $\left.Z_{(p)} \stackrel{x p}{\rightarrow} Z_{(p)} \rightarrow Z_{p}\right)$. From the computation of the 34 stem we see that $\alpha_{1} \alpha_{8}$ is not zero on $S^{5}$ and $\tilde{\alpha}_{9} h_{1}$ is born on $S^{3}$.

LEMMA (3.4). $\operatorname{Ext}^{0}(W(n)) \simeq F_{p}\left[u_{1}\right] x_{2 p n-1}$.

Proof. Suppose $x=\sum_{i} w_{i} x_{2 p^{i} n-1}\left(w_{i} \in A\right)$ is a cycle. Then from (2.5) we have

$$
0=d(x)=\sum_{i} d\left(w_{i}\right) \otimes x_{2 p^{i} n-1}-\sum_{i>1} w_{i}\left(\sum_{j<i} p^{i-j} h_{i-j}^{n p^{j}} \otimes x_{2 p^{j} n-1}\right) .
$$

Since $d\left(x_{2 p^{i} n-1}\right)$ is unstable we have

$$
0=\Sigma^{2} d(x)=\Sigma d\left(w_{i}\right) \otimes \Sigma^{2} x_{2 p^{i} n-1} .
$$

By [11] $w_{i}=\varepsilon_{i} u_{1}^{k_{i}}$. Since $2 p^{i} n-1 \geqslant 3, d\left(u_{1}^{k_{i}}\right) \otimes x_{2 p^{i} n-1}=0$ [4]. (3.5) now reduces to

$$
0=\sum_{i>1} w_{i} p^{i-j} h_{i-j}^{n p^{j}} \otimes x_{2 p^{i} n-1}
$$

By [3] it follows by induction that $w_{i} \equiv 0(\bmod p)$ for $i>1$.

LEMMA (3.6). $P_{2}\left(u_{1}^{k} x_{2 p n-1}\right)=0$ if and only if $k=s p^{t}-n$ with $n \leqslant t+1$.

PROof. The elements $\alpha_{s p t / n} \in \operatorname{Ext}_{\mathscr{Q}}^{1}(A(2 n+1))$ with $n \leqslant t+1$ generate $\operatorname{coker}\left\{\operatorname{Ext}_{\mathscr{Q}}^{1}(A(2 n-1)) \rightarrow \operatorname{Ext}_{\mathscr{Q}}^{1}(A(2 n+1))\right\}$ by (2.7). (3.6) now follows from the formula for the Hopf invariant given in (2.7).

COROLlaRy (3.7). The elements

$$
d\left(h_{1}^{s p^{t}}\right) \iota_{2 s p^{t}-1}, d\left(u_{1} h_{1}^{s p^{t}-1}\right) \iota_{2 s p^{t}-3}, \ldots, d\left(u_{1}^{s p^{t}-t-2} h_{1}^{t+2}\right) \iota_{2 t+3}
$$

are nonzero in the $\left(2(p-1) s p^{t}-2\right)$ stem. Furthermore, each element survives to an element of order $p$ in homotopy.

PROof. The fact that these elements are nonzero follows immediately from (2.5) and (3.6).

From the paragraph preceding (2.8) $P_{2}\left(u_{1}^{k} x_{2 p m-1}\right)$ is not zero exactly when $\alpha_{k} \in \pi_{2 p k+2 p n-2}\left(S^{2 p n+1}\right)$ is not a Hopf invariant. Let $H$ be the homotopy Hopf invariant

and

$$
H: \pi_{i+1}\left(\hat{S}^{2 n}\right) \rightarrow \pi_{i+1}\left(S^{2 n p-1}\right),
$$

$$
P: \pi_{i+1}\left(S^{2 n p+1}\right) \rightarrow \pi_{i+1}\left(\hat{S}^{2 n}\right)
$$


the map of $\S 1$. Then, as remarked in $\S 1, H P^{\prime}\left(\alpha_{k}\right)=0$ and $P^{\prime}\left(\alpha_{k}\right)$ desuspends to an element

$$
\lambda \in \pi_{2 P(n+k)-2(k-j)-1}\left(S^{2 n-1}\right) .
$$

$\lambda$ must be detected in filtration 2 since $P^{\prime}\left(\alpha_{k}\right)$ is detected in filtration 2 and no element of filtration 1 can suspend to an element of higher filtration. It follows from (3.4) that the only filtration 2 elements in this stem on the $(2 n-1)$ sphere which double suspends to zero is $P_{2}\left(u_{1}^{k} x_{2 p n-1}\right)$. This proves that the element survives to an element in the kernel of the double suspension map. They are of order $p$ in homotopy by Cohen, Moore and Neisendorfer [7].

The mod $p$ Whitehead product $P_{2}\left(x_{2 p n-1}\right)\left(n=s p^{t}, s \neq 0 \bmod p\right)$ has the form

$$
\begin{aligned}
d\left(h_{1}^{n}\right) & =s p^{t} h_{1} \otimes h_{1}^{n-1}+\sum_{i=2}^{n-1}\left(\begin{array}{c}
n \\
j
\end{array}\right) h_{1}^{i} \otimes h_{1}^{n-1} \\
& =s h_{1} u_{1}^{t} \otimes h_{1}^{n-t-1} \bmod S^{2(n-t-1)-1} ;
\end{aligned}
$$

the last equality follows from the fact that $p^{t+2-i} \mid\left(\begin{array}{c}s p^{t} \\ i\end{array}\right)$ if $1<i \leqslant t+1$ [14]. The following is an immediate consequence of the above computation.

Proposition (3.8). $P_{2}\left(x_{2 p n-1}\right)$ desuspends to $\gamma \in \operatorname{Ext}_{\mathscr{U}}\left(S^{2\left(s p^{t}-t-1\right)+1}\right)$ with $H_{2}(\gamma)$ $=s \tilde{\alpha}_{t+1}$.

Proof of (3.2). (i) Ext $=0$ in this case since the unstable cobar complex is zero in dimensions $\not \equiv 0 \bmod 2(p-1)$. (ii) (3.8) implies the bottom of the tower is correct. (3.7) implies the subscripts (i.e. the terms $u_{1}^{a} h_{1}^{t}$ in the notation of (3.1)) are correct. It remains to show that every entry in the tower desuspends as indicated. We prove this by induction starting from the top of the tower. We have the relation $u_{1}^{r} h_{1}^{s p^{t}-r}=$ $p^{r} h_{1}^{s p^{t}}+x$, where $x$ desuspends to $S^{2 z+1}$ with $z<s p^{t}-r$. We therefore have from (3.8)

$$
\begin{aligned}
d\left(u_{1}^{r} h_{1}^{s p^{t}-r}\right) & =p^{r} h_{1} u_{1}^{t} h_{1}^{s p^{t}-t-1}+d(x) \\
& =h_{1} u_{1}^{t+r} h_{1}^{s p^{t}-t-r-1}+d(x) \bmod S^{2\left(s p^{t}-t-r-1\right)-1} .
\end{aligned}
$$

Since $x$ desuspends to $S^{2 z+1}, d(x)$ must be homologous to a multiple of some element higher in the tower. In particular, $d(x)$ desuspends to $S^{2\left(s p^{t}-t-r-1\right)-1}$. This proves that each element desuspends at least as far as indicated. We cannot desuspend any further since, from (2.4),

$$
H_{2}\left(h_{1} u_{1}^{t+r} h_{1}^{s p^{t}-t-r-1} \otimes \iota_{2\left(s p^{t}-t-r-1\right)+1}\right)=h_{1} u_{1}^{t+r} x_{2\left(s p^{t}-t-r-1\right)-1}
$$

which is not zero in $\operatorname{Ext}_{\mathscr{Q}}^{1}\left(W\left(s p^{t}-t-r-1\right)\right)$. To see this we note that $h_{1} u_{1}^{t+s}$ is the stable $\bmod p$ reduction of $\tilde{\alpha}_{t+s+1}(2.7)$ which cannot be in the image of the $W$ coaction (2.5). To prove (iii) there are two cases.

Case I. $\alpha_{t+r}=\Sigma^{4} \gamma$ for $\gamma \in \operatorname{Ext}_{\mathscr{U}}^{1}\left(A\left(2 p\left(s p^{t}-t-r\right)-3\right)\right)$.

In this case we have enough suspensions for $\tilde{\alpha}_{t+r}$ to equal $h_{1} u_{1}^{n+r-1}$ on a $\bmod p$ class $x_{2 p\left(s p^{t}-t-r\right)-1}$. 
Case II. If $\tilde{\boldsymbol{\alpha}}_{t+r}$ is a double suspension but not a four-fold suspension with respect to $h_{1}^{s p^{t}-t-r} \otimes \iota_{2\left(s p^{t}-t-r\right)+1}$, then

$$
H_{2}\left(h_{1} u_{1}^{t+r-1} h_{1}^{s p^{t}-t-r} \otimes \iota_{2\left(s p^{t}-t-r\right)+1}\right)=h_{1} u_{1}^{t+r-1} \otimes x_{2 p\left(s p^{t}-t-r\right)-1} .
$$

Let $\beta$ be the Bockstein homomorphism induced in Ext by tensoring

$$
A\left(2 p\left(s p^{t}-t-r\right)-1\right)
$$

by the exact sequence $Z_{(p)} \stackrel{x p}{\rightarrow} Z_{(p)} \rightarrow Z_{p}$. Then $\beta\left(h_{1} u_{1}^{t+r-1}\right)=\alpha_{1} \alpha_{t+r-1} \cdot t+r$ must be $\equiv 0(\bmod p)$ so $\alpha_{1} \alpha_{t+r-1}$ is represented in the unstable cobar complex by

$$
h_{1} u_{1}^{t+r-2} h_{1} \otimes \iota_{2 p\left(s p^{t}-t-r\right)-1}
$$

mod lower spheres. This is the top of the tower in a lower stem. The suspension condition is equivalent to $\nu_{p}(t+r)+2=p\left(s p^{t}-t-r\right)$. (3.6) implies this element has not yet died and it follows that $h_{1} u_{1}^{t+r-1} \otimes x_{2 p\left(s p^{t}-t-r\right)}$ is not homologous to $\tilde{\alpha}_{t+r}$. There is also the unstable element

$$
p u_{1}^{a} h_{1}^{p\left(s p^{t}-t-r\right)} \otimes x_{2 p\left(s p^{t}-t-r\right)-1},
$$

where $a=p\left(s p^{t}-t-r\right)-(t+r)$. Since the exponent of $h_{1}$ is $\nu(t+r)+2$ it follows from part (ii) that $\beta\left(p u_{1}^{a} h_{1}^{p\left(s p^{t}-t-r\right)}\right)$ also equals $\alpha_{1} \alpha_{t+r-1}$. It follows that $h_{1} u_{1}^{t+r-2}-p u_{1}^{a} h_{1}^{p\left(s p^{t}-t-r\right)}$ is homologous to zero or $\tilde{\alpha}_{t+r}$. Since $h_{1} u_{1}^{t+r-2}-$ $p_{1} u^{a} h_{1}^{p\left(s p^{t}-t-r\right)}$ suspends nonzero it must be the latter. However, in $W$ we have

$$
d\left(u_{1}^{a} \otimes x_{2 p^{2}\left(s p^{t}-t-r\right)-1}\right)=p u_{1}^{a} h_{1}^{p\left(s p^{t-t-r)}\right.} \otimes x_{2 p\left(s p^{t}-t-r\right)-1}
$$

and $h_{1} u_{1}^{t+r-2}$ is homologous to $\tilde{\alpha}_{t+r}$ in $\operatorname{Ext}_{\mathscr{U}}(W)$.

The statement about the space of origin follows from (2.3). If the hypothesis of (iv) is satisfied, similar computations show that $h_{1} u_{1}^{t+r} \otimes x_{2 p\left(s p^{t}-t-r-1\right)-1}$ is not in the image of the map $I$ in (2.3) (see (3.3) for an example). The statement about the sphere of origin again follows from (2.3).

In order to prove $(v)$ we first consider towers in the $(2 s(p-1)-2)$ stem with $s \neq 0(\bmod p)$. (In the terminology of [17] these are elements of type I.) In this case there are no desuspensions and the elements in the tower are exactly those given in (3.7) proving (v) for $n=0$.

For $n>0$ we first note that the mod $p$ Whitehead product, $d\left(h_{1}^{s p^{n}}\right)$, desuspends in Ext exactly as far as it does in homotopy. (This is the mod $p$ vector fields problem and may be proven by the methods of [12, (III, 7.2)] using [10 and 17].) Let $\gamma$ be a maximal desuspension of $d\left(h_{1}^{s p^{n}}\right)$. Let $S^{2 t+1}$ be the sphere of origin for $\gamma$. Inductively assume that the generator $\lambda_{i}$ on $S^{2 i+1}$ survives $(2 \leqslant i \leqslant t)$. Since $n>0$, $p \lambda_{i} \neq 0$. By [7] $p \lambda_{i}$ desuspends in homotopy to an element which must be detected in filtration 2 by $\lambda_{i-1}$, the generator of the tower on $S^{2 i-1}$.

To see that there can be no extensions we first consider the elements on $S^{2 j+1}$, $j \leqslant t+1$. The generator of the tower on this sphere is of order $p^{j}$. There can be no higher extensions by [7 and 16]. From the $(2 t+5)$ sphere on the elements of order $p$ in Ext are the elements given in (3.7) which survive to elements of order $p$ in homotopy. Hence these are no higher $B P$ extensions. 
4. Applications. The products of elements from the one line are stably zero [14, (8.18)]; they therefore represent potential unstable elements. In fact, in the metastable range, when they are first created they are nonzero. In order to understand when they die it suffices to identify each composition in the unstable tower.

For the composition

$$
\alpha_{s p^{n} / i} \alpha_{r p^{m} / j} \otimes \iota_{2 k+1}
$$

in Ext to make sense in homotopy we must have $j \leqslant k$ and $i \leqslant k+(p-1) r p^{m}-1$, i.e. $\alpha_{s p^{n} / i}$ is a double suspension with respect to $\alpha_{r p^{m} / j} \otimes \iota_{2 k+1}$ in Ext.

For the following theorem we assume the stable conditions: $i \leqslant n+1, j \leqslant m+1$ and a metastable condition: $\alpha_{r p^{n} / n+1}$ is defined on $\alpha_{r p^{m} / j} \otimes \iota_{2 j+1}$ in homotopy. This condition simplifies the computation and includes all cases needed for the sequel.

THEOREM (4.1). $\alpha_{s p^{n} / i} \alpha_{r p^{m} / j} \otimes \iota_{2 k+1}$;

(i) $=0$ if $i+j \leqslant n+1$;

(ii) if $i+j>n+1,=0$ if $k \geqslant \nu\left(s p^{n}+r p^{m}\right)+i+j-n$;

(iii) in the situation of (ii) the composition desuspends to the $(2(i+j-n-1)+1)$ sphere with $\mathrm{H}_{2}$ given by

$$
\begin{array}{ll}
\tilde{\alpha}_{\left(s p^{n}+r p^{m}-n-i-j-1\right)} & \text { if } \nu\left(s p^{n}+r p^{m}-n-i-j+1\right) \leqslant p(i+j-n-1)-1, \\
h_{1} u_{1}^{s p^{n}+r p^{m}-n-i-j} & \text { otherwise. }
\end{array}
$$

Proof. The metastable condition guarantees that

$$
\alpha_{s p^{n} / n+1} \alpha_{r p^{m} / j} \otimes \iota_{2 j+1}=\alpha_{s p^{n} / n+1} \otimes u_{1}^{r p^{m}-j} h_{1}^{j} \otimes \iota_{2 j+1}
$$

mod terms which desuspend.

From (2.4),

$$
H_{2}\left(\alpha_{s p^{n} / n+1} \alpha_{r p^{m} / j} \otimes \iota_{2 j+1}\right)=\alpha_{s p^{n} / n+1} u_{1}^{r p^{m}-j} \otimes x_{2 j+1} .
$$

$\alpha_{s p^{n} / n+1} u_{1}^{r p^{m}-j}$ suspends to $h_{1} u_{1}^{\left(s p^{n}-1\right)+\left(r p^{m}-j\right)}$ which is nonzero $\bmod p$. Since $\alpha_{s p^{n} / n+1} u_{1}^{r p^{m}-j}$ is stably nonzero it is nonzero in $\operatorname{Ext}_{\mathscr{U}}(W(j))$. Since on each sphere there is at most one new unstable element it follows that $\alpha_{s p^{n} / n+1} \alpha_{r p^{m} / j} \otimes \iota_{2 j+1}$ is (up to a unit) the generator of the tower on the $(2 j+1)$ sphere. From (3.2) it follows that this element is nonzero for exactly $\nu\left(s p^{n}+r p^{m}\right)$ double suspensions, proving (4.1) when $i=n+1$. For the general case we have

$$
\alpha_{s p^{n} / i} \alpha_{r p^{m} / j}=p^{n+1-i} \alpha_{s p^{n} / n+1} \alpha_{r p^{m} / j}=d\left(p^{n+1-i} x\right),
$$

where $x$ is the subscript which kills $\alpha_{s p^{n} / n+1} \alpha_{r p^{m} / j}$. If $i+j \leqslant n+1$ the composition has been desuspended to the 1 sphere and is zero (on $S^{1}$ ). If $i+j>n+1$, $\alpha_{s p^{n} / i} \alpha_{r p^{m} / j} \otimes \iota_{2(i+j-n-1)+1}$ has a nonzero $H_{2}$ and does not die until the sphere indicated. The $\mathrm{H}_{2}$ computation in (iii) corresponds to (3.2)(iii) and (iv).

Corollary (4.2). The mod $p$ Whitehead product $\in \pi_{2 p n-3}\left(S^{2 n-1}\right)$ is indecomposable except for $\alpha_{1}^{2} \in \pi_{4 p-3}\left(S^{3}\right)$ and, if $p=3, \alpha_{2} \alpha_{1} \in \pi_{15}\left(S^{5}\right)$.

Proof. For dimension reasons the metastable condition is satisfied. Since the Whitehead product appears in filtration 2 it must be a composite of elements from 
the 1 line (modulo $B P$-filtration). The composition $\alpha_{s p^{n} / i}{ }^{\circ} \alpha_{r p^{m} / j}$ dies on $S^{2 k+1}$, where $k=\nu\left(s p^{n}+r p^{m}\right)+i+j-n$. On the other hand, the $\bmod p$ Whitehead product in this stem dies on $S^{2\left(s p^{n}+r p^{m}\right)+1}$. These two numbers are equal only in the cases indicated.

We now consider certain sphere bundles over spheres which were studied in [15 and 8]. As usual $p$ is an odd prime.

$$
B_{n}(p)=S^{2 n+1} \cup_{\alpha_{1}} e^{2 n+2 p-1} \cup e^{4 n+2 p}
$$

with cohomology ring

$$
H^{*}\left(B_{n}(p) ; Z_{(p)}\right) \simeq \Lambda\left(y_{2 n+1}, y_{2 n+2 p-1}\right) .
$$

The $E_{2}$ term of the unstable Novikov spectral sequence for $B_{n}(p)$ is therefore given by $\operatorname{Ext}_{\mathscr{U}}\left(B P_{*}\left\{w_{2 n+1}, w_{2 n+2 p-1}\right\}\right)$, where $\psi\left(w_{2 n+2 p-1}\right)=1 \otimes w_{2 n+2 p-1} c+h_{1}$ $\otimes w_{2 n+1}$. We have a fibration [15]

$$
S^{2 n+1} \rightarrow B_{n}(p) \rightarrow S^{2 n+2 p-1}
$$

which induces a long exact sequence in homotopy and in Ext:

$$
\begin{aligned}
& \rightarrow \operatorname{Ext}_{\mathscr{U}}^{s}(A(2 n+1)) \rightarrow \operatorname{Ext}_{\mathscr{U}}^{2}\left(B p_{*}\left\{w_{2 n+1}, w_{2 n+2 p-1}\right\}\right) \\
& \rightarrow \operatorname{Ext}_{\mathscr{U}}^{s}(A(2 n+2 p-1)) \stackrel{\partial}{\rightarrow} \operatorname{Ext}_{\mathscr{U}}^{s+1} \rightarrow
\end{aligned}
$$

with $\partial(\beta)=\beta \alpha_{1}$.

While $\partial$ is given by composition with $\alpha$ in Ext it is not composition with $\alpha_{1}$ in homotopy unless $\beta$ is a double suspension. (Hence if $\beta$ is not a double suspension, $\partial(\beta)$ is not a nonmetastable example of (4.1).) For example, we have for $p=3$ the fibration $S^{3} \rightarrow B_{1}(3) \rightarrow S^{7}$ with $\alpha_{9 / 3} \in \operatorname{Ext}_{\mathscr{Q}}^{1}(A(7))$ not a double suspension. $\partial\left(\alpha_{9 / 3}\right)=\alpha_{9 / 3} \alpha_{1}$ on $S^{3}$ in Ext but this composition is not defined in homotopy. By [3, (5.3ii)] $\alpha_{9 / 3} \alpha_{1}$ must be homologous to a class which is defined in homotopy (see (4.10)).

From (4.4) and [7] we see that the exponent of $\pi_{*}\left(B_{n}(p)\right)$ is less than or equal to $p^{2 n+p-1}$.

We now consider the question of realizing $p^{2 n+p-1}$ by studying the $E_{2}$ exponent.

We first show that the exponent in $E_{2}$ is $<p^{2 n+p-1}$ if the bottom attaching map in $B_{n}(p)$ is a double suspension.

Proposition (4.6). $B P_{*}\left(\Omega B_{n}(p)\right) \simeq P\left(y_{2 n}, y_{2 n+2 p-2}\right)$.

Proof. The fibration

$$
\Omega S^{2 n+1} \rightarrow \Omega B_{n}(p) \rightarrow \Omega S^{2 n+2 p-1}
$$

homologically splits [15]. (4.6) now follows from the Atiyah-Hirzebruch spectral sequence.

Proposition (4.7). There is a long exact sequence

$$
\rightarrow E_{2}^{s}\left(B_{n}(p)\right) \rightarrow E_{2}^{s}\left(B_{n+1}(p)\right) \rightarrow \operatorname{Ext}_{\mathscr{U}}^{s-1}(Q(n)) \rightarrow E_{2}^{s+1} \rightarrow,
$$

where $Q(n)$ is a $\left(B P_{*} / p\right)$-module. 
Corollary (4.8). (i) The exponent of $E_{2}\left(B_{n}(p)\right)$ for $n>1$ is less than $p^{2 n+p-1}$.

(ii) $E_{2}^{1}\left(B_{n}(p)\right) \rightarrow E_{2}^{1}\left(B_{n+1}(p)\right)$ is 1-1.

Proof of (4.7). Consider the composite functor spectral sequence converging to $E_{2}^{*}\left(\Omega B_{n+1}(p)\right)[5]$ :

$$
\operatorname{Ext}_{\mathscr{L}}^{*}\left(R^{*} B P_{*}\left(\Omega B_{n+1}(p)\right)\right) \Rightarrow E_{2}^{*}\left(\Omega B_{n+1}(p)\right),
$$

where $R^{*} P$ is the ${ }^{*}$ th derived functor of the primitives. As $B P_{*}$-modules

$$
R^{*} P B P_{*}\left(\Omega B_{n+1}(p)\right) \simeq R^{*} P B P_{*}\left(\Omega S^{2 n+1}\right) \oplus R^{*} P B P_{*}\left(\Omega S^{2 n+p-1}\right) .
$$

$R^{*} P B P_{*}\left(\Omega S^{2 k+1}\right)$ has been computed in [6];

$$
R^{*} P B P_{*}\left(\Omega S^{2 k+1}\right)= \begin{cases}B P_{*}\left(S^{2 k}\right) & \text { for } i=0 \\ W(k) & \text { for } i=1 \\ 0 & \text { for } i>1\end{cases}
$$

where $W(k)$ is a $Z_{p}$ vector space. The composite functor spectral sequence now reduces to

$$
\rightarrow \operatorname{Ext}_{\mathscr{U}}^{s}\left(P B P_{*}\left(\Omega B_{n+1}(p)\right)\right) \rightarrow E_{2}^{s}\left(\Omega B P_{n+1}(p)\right) \rightarrow \operatorname{Ext}_{\mathscr{U}}^{s-1}(Q(n)) \rightarrow
$$

where $Q(n) \simeq W(n) \oplus W(n+p-1)$ is a vector space. The definition of $U$ given in (2.1) immediately implies $\operatorname{Ext}_{\mathscr{Q}}^{s}\left(P B P_{*}\left(\Omega B_{n+1}(p)\right)\right) \simeq E_{2}^{s}\left(B_{n}(p)\right)$. An argument similar to the proof of [6, Theorem (6.1)] shows that $E_{2}^{s}\left(\Omega B_{n+1}(p)\right) \simeq E_{2}^{s}\left(B P_{n+1}(p)\right)$. Substituting this isomorphism into (4.9) proves (4.7).

REMARK. (i) Corollary (4.8) does not prove the exponent of $\pi_{*}\left(B_{n}(p)\right)$ is < $p^{2 n+p-1}$ if $n>1$ since there may be extensions. We conjecture this cannot happen.

(ii) A result similar to (4.7) is true for spaces constructed in [8] with an arbitrary element of $\operatorname{Im} J$ as bottom attaching map.

We now restrict our attention to $B_{1}(p)(=B) . B$ is an $S^{3}$ bundle over $S^{2 p+1}$. In order to construct elements of order $p^{p+1}$ in $E_{2}$ (and $\pi_{*}$ ) it is natural to try to pull the desuspension of the stable elements in $\operatorname{Im} J$ of order $p^{p}$ back to $B$.

We require the following $B P$-formulas:

(4.10) Modulo classes defined in $S^{1}$ :

$$
\begin{aligned}
& 1 \cdot u_{2}=u_{2}+u_{1}^{p} h_{1}-h_{1}^{p} \cdot u_{1} \bmod p \text { on } S^{3}, \\
& \psi\left(h_{2}\right)=h_{2} \otimes 1+1 \otimes h_{2}+h_{1}^{p} \otimes h_{1} .
\end{aligned}
$$

THEOREM (4.11). Let $t \geqslant p-1$. Then $\partial\left(\alpha_{s p^{t} / p}\right) \neq 0$ unless $t=p-1$ and $s=$ $(-1)^{s+1} \bmod p$.

Proof. Modulo terms defined on $S^{1}$, we have, from (2.7),

$$
\partial\left(\alpha_{s p^{t} / p}\right)=\alpha_{s p^{t} / p} \alpha_{1} \iota_{3}=\left\{\begin{array}{l}
(-1)^{s} p^{s p^{t}-p} h_{1}^{s p^{t}} \otimes h_{1} \otimes \iota_{3} \text { if } t>p-1, \\
(-1)^{s} p^{s p^{t}-p} h_{1}^{s p^{t}} \otimes h_{1} \otimes \iota_{3}-s h_{1} u_{1}^{s p^{t}-1} \otimes h_{1} \otimes \iota_{3} \\
\text { if } t=p-1 .
\end{array}\right.
$$


By adding $d\left((-1)^{s} p^{s p^{t}-p} h_{1}^{s p^{t}-p} h_{2} \otimes \iota_{3}\right)$ to (4.12) we have mod $S^{1}$

$$
x=(-1)^{s} p^{s p^{t}-p} h_{1}^{s p^{t}} \otimes h_{1} \otimes \iota_{3} \sim-(-1)^{s} h_{1}^{p} u_{1}^{s p^{t}-p} \otimes h_{1} \otimes \iota_{3} .
$$

By subtracting $d\left((-1)^{s} u_{2} u_{1}^{s p^{t}-p-1} h_{1} \otimes \iota_{3}\right)$, using (4.10) and the fact that we have enough room to pass $u_{1}$ 's ro the right past $h_{1}$ 's we obtain

$$
x \sim-(-1)^{s} u_{1}^{s p^{t}-1} h_{1} \otimes h_{1} \otimes \iota_{3} .
$$

We now have

$$
\partial\left(\alpha_{s p^{t} / p}\right)=\left\{\begin{array}{l}
-(-1)^{s+1} u_{1}^{s p^{t}-1} h_{1} \otimes h_{1} \otimes \iota_{3} \text { if } t>p-1 \\
(-1)^{s+1} u_{1}^{s p^{t}-1} h_{1} \otimes h_{1} \otimes \iota_{3}-s h_{1} u_{1}^{s p^{t}-1} \otimes h_{1} \otimes \iota_{3} \quad \text { if } t=p-1 .
\end{array}\right.
$$

From (3.2) we know $\partial\left(\alpha_{s p^{t} / p}\right)$ is zero if and only if $H_{2}\left(\partial \alpha_{s p^{t} / p}\right)$ is stably zero. If $t>p-1$, then $H_{2}= \pm h_{1} u_{1}^{s p^{p}-1}$ stably which is not zero mod $p$. If $t=p-1$, then $H_{2}=\left((-1)^{s+1}-s\right)\left(h_{1} u_{1}^{s p^{t}-1}\right)$ stably. This is zero if and only if $s \equiv(-1)^{s+1} \bmod p$.

(4.11) resolves one of the ambiguous cases in [15, (6.4)]. In Oka's notation A, $(1,39) \simeq Z_{3^{4}}$, generated by $\left[\alpha_{9}^{\prime}(7)\right](p=3)$. Hence the maximal exponent is realized in homotopy. The ambiguous extension in [15, p. 191] may also be resolved by direct computation to give $\left[\gamma_{3}(7)\right]$ generates a $Z_{3^{4}}\left(\mathrm{Oka}\right.$ 's $\gamma_{3}(7)$ is the element $\left(\tilde{\alpha}_{6} h_{1}^{3}\right)_{u_{1}^{3} h_{1}^{6}}$ in the tower).

The extensions in $E_{2}(B)$ can be determined using the $\bar{e}$ invariant defined in [2, (4.3)] which extends the Adams $e$ invariant to $C^{1}(A(2 n+1))$.

Let $[\gamma]$ denote a pull-back of $\gamma \in E_{2}\left(s^{2 n+2 p-1}\right)$ to $E_{2}(B)$.

Proposition (4.13). Let $s \equiv(-1)^{s+1} \bmod p$. Then $\left[\alpha_{s p^{p-1} / p}\right]$ generates $a Z_{p^{p+1}}$.

Proof. From the proof of (4.11) there is an $x$ with $y=\partial\left(\alpha_{s p^{p-1} / p}\right)+d(x)$ desuspending to $S^{1}$ and $\bar{e}(x)=n / p^{2} . y=d(z), z$ defined on $S^{1}$ and therefore $\bar{e}(z) \in Z$. It follows that

$$
\left[\alpha_{s p^{p-1} / p}\right]=\alpha_{s p^{p-1} / p} \otimes x_{2 n+2 p-1}+w \otimes x_{3},
$$

where $\bar{e}(w)=m / p^{2}$.

$$
p^{p}\left[\alpha_{s p^{p-1} / p}\right]=\left(-u_{1}^{s p^{p-1}} h_{1}+p^{p} w\right) \otimes x_{3} .
$$

$\bar{e}$ of this cycle is $1 / p$ and hence is homologous to $\alpha_{s p^{p-1}+1}$.

\section{BIBLIOGRAPHY}

1. J. F. Adams, Stable homotopy and generalized homology, University of Chicago Press, Chicago, 1974.

2. M. Bendersky, Some computations in the unstable Adams-Novikov spectral sequence, Publ. Res. Inst. Math. Sci. 16 (1980), 739-766.

3. 599-619.

4. The BP Hopf invariant (to appear).

5. M. Bendersky, E. B. Curtis and H. R. Miller, The unstable Adams spectral sequence for generalized homology, Topology, 17 (1978), 229-248.

6. M. Bendersky, E. B. Curtis and D. C. Ravenel, The EHP sequence in BP-theory, Topology 21 (1982), 373-391. 
7. F. R. Cohen, J. C. Moore and J. A. Neisendorfer, The double suspension and exponents of the homotopy groups of spheres, Ann. of Math., (2) 110 (1979), 549-565.

8. G. Cooke, J. R. Harper and A. Zabrodsky, Torsion free mod $p$ H-spaces of low rank, Topology 18 (1979), 349-359.

9. B. Gray, On the sphere of origin of infinite families in the homotopy groups of spheres, Topology 8 (1969), 219-232.

10. T. Kambe, H. Matsumaga and H. Toda, A note on stunted lens space, J. Math. Kyoto Univ. 5 (1966), 143-149.

11. P. S. Landweber, Invariant regular ideals in Brown-Peterson homology, Duke J. Math. 42 (1975), 499-505.

12. M. E. Mahowald, The meta-stable homotopy of $S^{n}$, Mem. Amer. Math. Soc. No. 72, 1967.

13. The image of $J$ in the EHP sequence, Ann. of Math. (2) 116 (1982), 65-112.

14. H. R. Miller, D. C. Ravenel and W. S. Wilson, Periodic phenomena in the Adams-Novikov spectral sequence, Ann. of Math. (2) 106 (1977), 469-516.

15. S. Oka, On the homotopy groups of sphere bundles over spheres, J. Sci. Hiroshima Univ. 33 (1969), $161-195$.

16. P. S. Selick, Odd primary torsion in $\pi_{k}\left(S^{3}\right)$, Topology 17 (1978), 407-412.

17. H. Toda, On iterated suspensions. I, II, J. Math. Kyoto Univ. 5 (1965), 87-142; 5 (1966), 209-250.

18. B. Gray, Unstable families related to the image of $J$ (to appear).

Department of Mathematics, Rider College, LaWrenceville, NeW Jersey 08648 